\title{
SÍNTESE, CARACTERIZAÇÃO E AVALIAÇÃO DA ATIVIDADE CITOTÓXICA DE COMPOSTOS CICLOPALADADOS CONTENDO COMO LIGANTES BENZALDEIDOXIMA ORTOMETALADA E ETILENOTIOURÉIA
}

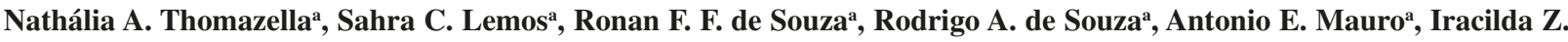 \\ Carlos $^{\mathrm{b}}$ e Adelino V. G. Netto ${ }^{\mathrm{a}, *}$ \\ aDepartamento de Química Geral e Inorgânica, Instituto de Química, Universidade Estadual Paulista, 14800-060 Araraquara - SP, \\ Brasil \\ bepartamento de Análises Clínicas, Faculdade de Ciências Farmacêuticas, Universidade Estadual Paulista, 14800-903 Araraquara \\ - SP, Brasil
}

Recebido em 08/06/2018; aceito em 03/09/2018; publicado na web em 25/09/2018

\begin{abstract}
SYNTHESIS, CHARACTERIZATION AND CYTOTOXIC ACTIVITY EVALUATION OF CYCLOPALLADATED COMPOUNDS CONTAINING ORTHOMETALLATED BENZALDEHYDEOXIME AND ETHYLENETHIOUREA AS LIGANDS. The halidebridge cleavage reaction of the $\left[\operatorname{Pd}\left(C^{2}, N \text {-bzox }\right)(\mu-\mathrm{Cl})\right]_{2}$ compound (bzox = benzaldehydeoxime) with ethylenethiourea $(\mathrm{L})$ in the $1: 2$ molar ratio at room temperature yielded the cyclopalladated compound $\left[\mathrm{Pd}\left(C^{2}, N\right.\right.$-bzox $\left.)(\mathrm{Cl})(\mathrm{L})\right](\mathbf{1})$ whose replacement of the halide group by the addition of suitable salts afforded compounds of general formulae $\left[\mathrm{Pd}\left(C^{2}, N-\mathrm{bzox}\right)(\mathrm{X})(\mathrm{L})\right]\left\{\mathrm{X}=\mathrm{Br}(\mathbf{2}), \mathrm{I}^{-}(\mathbf{3})\right\} . \mathrm{All}$ compounds were characterized by melting point, elemental analyses, infrared and ${ }^{1} \mathrm{H}$ - and ${ }^{13} \mathrm{C}\left\{{ }^{1} \mathrm{H}\right\}$-NMR spectroscopies revealing the complexes formation with the oxime coordinated to $\mathrm{Pd}(\mathrm{II})$ as $C, N$-chelating and the ethylenethiourea as a monodentate ligand via the sulfur atom. The cytotoxicity of cyclopalladated compounds has been evaluated in vitro against murine mammary tumor cell (LM3) showing cytotoxic activity similar to cisplatin.
\end{abstract}

Keywords: cyclopalladated complexes; oximes; ethylenethiourea; imidazolidine-2-thione; cytotoxicity.

\section{INTRODUÇÃO}

Os complexos metálicos têm sido extensivamente investigados por apresentarem propriedades antitumorais que configuram um novo horizonte para o desenvolvimento de novos fármacos. ${ }^{1-4} \mathrm{~A}$ incorporação de metais de transição nas estruturas de moléculas candidatas a fármacos pode conferir-lhes propriedades inusitadas com relação aos seus análogos puramente orgânicos, como acidez de Lewis, magnetismo, atividade redox, luminescência, entre outros. Além disso, os íons metálicos apresentam números de coordenação, preferências estereoquímicas e reatividade que dependem da sua carga, tamanho e estrutura eletrônica, tornando-os uma plataforma única para o planejamento de novos fármacos com conceitos muito diferentes daqueles usados para moléculas orgânicas. ${ }^{5,6}$ Atualmente, fármacos como a cisplatina, carboplatina e oxaliplatina são utilizados em cerca de 50 a $70 \%$ dos tratamentos de tumores, ${ }^{7}$ contudo, a baixa seletividade unida à resistência adquirida pelo organismo a essas drogas e aos efeitos colaterais que elas causam motivam o desenvolvimento de novos compostos mais eficazes e menos tóxicos. ${ }^{8,9}$ Nesse contexto, os complexos de paládio(II) representam uma alternativa promissora pelas semelhanças entre os íons $\mathrm{Pd}(\mathrm{II})$ e Pt(II). No entanto, devido à alta labilidade desses complexos em solução, diferentes estratégias são empregadas para aumentar sua estabilidade, possibilitando assim que a integridade estrutural do complexo seja mantida até que o(s) alvo(s) farmacológico(s) seja(m) atingido(s).

Alicerçados em argumentos da Química de Coordenação, os químicos inorgânicos sintéticos logo desenvolveram uma estratégia para obter complexos de Pd(II) biologicamente ativos e mais estáveis do ponto de vista cinético utilizando ligantes quelantes. ${ }^{5,10,11}$ Dentre os tipos de compostos quelatos de paládio(II), os ciclopaladados ocupam uma posição de destaque por apresentarem: i) atividade

\footnotetext{
*e-mail: adelino.netto@unesp.br
}

citotóxica contra células tumorais sensíveis e resistentes à cisplatina; ii) capacidade de diminuir o crescimento de tumores em modelos de animais; iii) atividade antiproliferativa potente frente à microorganismos patógenos. ${ }^{12-17}$

Ciclopaladado pode ser definido como qualquer composto quelato de paládio que contenha uma ligação $\mathrm{Pd}-\mathrm{C}$, estabilizada intramolecularmente por um ou dois átomos doadores neutros (Y), pertencentes aos grupos 15 ou 16 da tabela periódica. ${ }^{18}$ Nas últimas décadas, compostos ciclopaladados vêm suscitando cada vez mais interesse da comunidade científica pelas suas potencialidades medicinais. ${ }^{16-17}$ Como exemplo, a citotoxicidade do composto ciclopaladado $\left[\mathrm{Pd}_{2}\left(\mathrm{R}_{(+)} C^{2}, N \text {-dmpa }\right)_{2}(\mu\right.$-dppe $\left.) \mathrm{Cl}_{2}\right],\left\{\mathrm{R}_{(+)} C^{2}, N\right.$-dmpa $=N, N$-dimetil1 -feniletilamina e dppe $=1,2$-etanobis(difenilfosfina) $\}$ frente à linhagem de leucemia humana (K562) foi investigada por Moraes et $a l$, sendo demonstrada que a atividade citotóxica, in vitro, inferior a $1,50 \mu \mathrm{mol} \mathrm{L} \mathrm{L}^{-1}$, estava associada à oxidação de proteínas presentes na membrana mitocondrial, além do fato do composto ser capaz de promover a morte das células tumorais sem apresentar toxicidade para células sanguíneas normais. ${ }^{19}$

A incorporação de tiouréias no arcabouço molecular de ciclopaladados tem se mostrado uma estratégia interessante na obtenção de compostos biologicamente ativos. Nesse contexto, Moro et al. investigaram a atividade citotóxica dos ciclometalados $\left[\mathrm{Pd}\left(C^{2}, N\right.\right.$-dmba) $\left.(\mathrm{X})(\mathrm{L})\right]$, $\left(\mathrm{X}=\mathrm{Cl}^{-}, \mathrm{Br}, \mathrm{I} ; C^{2}, N\right.$-dmba $=N, N$-dimetilbenzilamina, $\mathrm{L}=$ tiouréia $)$ frente à linhagem de células de adenocarcinoma mamário murino (LM3). ${ }^{20,21} \mathrm{O}$ complexo sintetizado $\left[\mathrm{Pd}\left(\mathrm{C}^{2}, \mathrm{~N}-\mathrm{dmba}\right)(\mathrm{I})(\mathrm{L})\right]$ apresentou um potencial citotóxico $\left(\mathrm{IC}_{50}=14,4 \pm 1,92 \mathrm{~mol} \mathrm{~L}^{-1}\right)$ frente à linhagem $\mathrm{LM} 3$ mais ativo que a cisplatina $\left(\mathrm{IC}_{50}=30,3 \pm 3,72 \mu \mathrm{mol} \mathrm{L}{ }^{-1}\right) .{ }^{9} \mathrm{Cabe}$ destacar que os ciclopaladados demonstraram não ser genotóxicos, mostrando que os mesmos apresentam um mecanismo de ação que não envolve necessariamente a interação com o DNA.

De acordo com Higgins et al., ${ }^{22}$ compostos de paládio portadores de iminas ortometaladas $\left(\mathrm{R}^{\prime \prime} \mathrm{R}^{\prime} \mathrm{C}=\mathrm{NR}\right)$ tendem a ser mais 
citotóxicos que aqueles constituídos por aminas alifáticas, como a $N, N$-dimetilbenzilamina (dmba). Apesar dos avanços significativos nessa área, ainda não há relatos na literatura sobre os efeitos provocados na citotoxicidade dos ciclopaladados contendo oximas ortometaladas.

Oximas representam uma classe de compostos orgânicos de fórmula R' $\mathrm{R}$ ' $\mathrm{C}=\mathrm{NOH}$ formadas pela condensação entre aldeído/cetona e a hidroxilamina. ${ }^{23}$ Ciclopaladados contendo oximas ortometaladas têm sido amplamente estudados por suas propriedades catalíticas em diversas reações orgânicas, sendo muito utilizados como catalisadores em reações de Heck e Suzuki-Miyaura. ${ }^{24}$ Embora as propriedades catalíticas dessa classe de ciclopaladados tenha motivado um número significativo de estudos, suas propriedades biológicas permanecem ainda inexploradas.

Dando continuidade ao interesse do nosso grupo pelas relações estrutura-atividade envolvendo compostos de paládio, ${ }^{25-28}$ e partindo do nosso conhecimento prévio a respeito da estrutura e atividade dos compostos $\left[\mathrm{Pd}\left(C^{2}, N\right.\right.$-dmba $\left.)(\mathrm{X})(\mathrm{L})\right]$, cuja citotoxicidade já é bem estabelecida, ${ }^{20,21}$ esse trabalho tem como objetivo investigar a influência da natureza do anel ciclometalado na citotoxicidade dessa classe de compostos por meio da síntese de uma classe de compostos análogos de fórmula $\left[\operatorname{Pd}\left(C^{2}, N\right.\right.$-bzox $\left.)(\mathrm{X})(\mathrm{L})\right]\left\{C^{2}, N\right.$-bzox = benzaldeidoxima, $\mathrm{L}=$ etilenotiouréia; $\mathrm{X}=\mathrm{Cl}^{-}(\mathbf{1}), \mathrm{Br}^{-}(\mathbf{2})$ e $\left.\mathrm{I}^{-}(\mathbf{3})\right\}$, visando averiguar o efeito da substituição do anel ortometalado na citotoxicidade dos compostos obtidos, como indicado no Esquema 1.

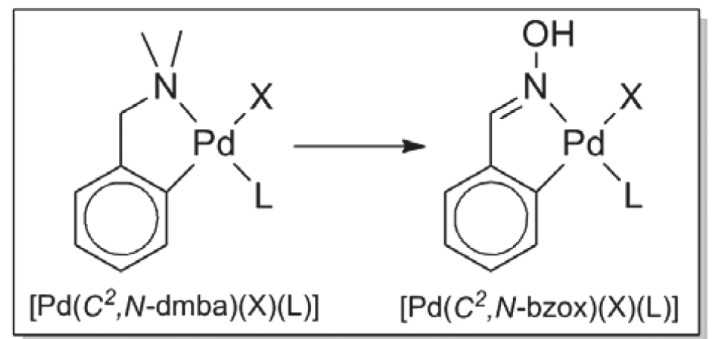

Esquema 1. Modificação no anel ciclometalado do composto ativo $\left[P d\left(C^{2}, N\right.\right.$ $-d m b a)(X)(L)]$

\section{PARTE EXPERIMENTAL}

\section{Materiais}

Os reagentes $E$-benzaldeidoxima (Aldrich), $N, N$-dimetilbenzilamina (Aldrich), cloreto de paládio(II) (Aldrich), etilenotiouréia (Aldrich), brometo de potássio (Merck) e iodeto de potássio (Merck) foram utilizados sem purificação adicional. Os solventes acetona, metanol, clorofórmio, acido acético e $n$-pentano de pureza analítica foram adquiridos da Merck.

\section{Caracterização dos complexos 1-3}

As análises quantitativas de carbono, hidrogênio e nitrogênio foram realizadas no analisador elementar Perkin Elmer, modelo 2400 Series II CHNS-O. Os espectros de absorção na região do infravermelho foram registrados no espectrofotômetro NICOLET, modelo SX-FT Impact 400, na região de 4000-400 $\mathrm{cm}^{-1}$, utilizando a técnica de pastilha de $\mathrm{KBr}$. Os espectros de ressonância magnética nuclear de ${ }^{1} \mathrm{H} \mathrm{e}{ }^{13} \mathrm{C}\left\{{ }^{1} \mathrm{H}\right\}$ foram registrados em espectrômetro multinuclear VARIAN, modelo INOVA 500, operando a $500 \mathrm{MHz}$ para hidrogênio. Acetona deuterada (Aldrich) foi utilizada para dissolução dos complexos enquanto que dimetilsulfóxido deuterado (Aldrich) para a dissolução dos ligantes (benzaldeidoxima e etilenotiouréia) livres.

\section{Síntese dos complexos 1-3}

O dímero $\left[\operatorname{Pd}\left(C^{2}, N-d m b a\right)(\mu-C l)\right]_{2}$ foi obtido, previamente, por meio da reação entre cloreto de paládio(II) e $N, N^{\prime}$-dimetilbenzilamina (dmba), como descrito na literatura. ${ }^{29-31} \mathrm{O}$ complexo precursor $\left[\operatorname{Pd}\left(C^{2}, N \text {-bxoz }\right)(\mu-C l)\right]_{2},(\mathbf{P})$ foi sintetizado pela reação de transciclometalação entre o dímero $\left[\mathrm{Pd}\left(C^{2}, N \text {-dmba }\right)(\mu-\mathrm{Cl})\right]_{2}$ e a benzaldeidoxima livre. ${ }^{32,33}$

Os complexos $\left[\mathrm{Pd}\left(C^{2}, N\right.\right.$-bzox $\left.)(\mathrm{X})(\mathrm{L})\right]\left\{\mathrm{X}=\mathrm{Cl}^{-}(\mathbf{1}), \mathrm{Br}(\mathbf{2})\right.$ e I- $(\mathbf{3})$; $\mathrm{L}=$ etilenotiouréia $\}$ foram obtidos por metodologia semelhante à descrita por Moro et al. ${ }^{21,22}$ Resumidamente, 4,0 mL de uma solução metanólica contendo etilenotiouréia $(0,380 \mathrm{mmol})$ foi adicionada, gota a gota, ao $\left[\mathrm{Pd}\left(C^{2}, N \text {-bxoz }\right)(\mu-\mathrm{Cl})\right]_{2}(0,190 \mathrm{mmol})$ suspenso em $10,0 \mathrm{~mL}$ de uma mistura de metanol:acetona 1:1. A solução resultante foi mantida em agitação magnética por $1 \mathrm{~h}$, à temperatura ambiente. Posteriormente, o solvente foi removido sob pressão reduzida e o sólido amarelado resultante foi lavado com metanol e $n$-pentano e seco sob vácuo resultando no complexo 1. Rendimento: $54 \%$.

Os complexos $\mathbf{2}$ e $\mathbf{3}$ foram obtidos adicionando 3,0 $\mathrm{mL}$ de uma solução metanólica de $\mathrm{KBr}$ ou KI (0,190 mmol), contendo 15 gotas de água, ao $\left[\mathrm{Pd}\left(C^{2}, N\right.\right.$-bzox $\left.)(\mathrm{Cl})(\mathrm{L})\right](0,190 \mathrm{mmol})$ solubilizado em 10,0 $\mathrm{mL}$ de uma mistura de metanol:acetona 1:1. A solução resultante foi mantida em agitação magnética por $1 \mathrm{~h}$, a temperatura ambiente, e o solvente foi removido sob pressão reduzida. Os sólidos obtidos foram lavados com água e $n$-pentano e secos sob vácuo. Rendimento: $95 \%$.

\section{Linhagem celular e meio de cultura}

A linhagem tumoral murina de adenocarcinoma mamario (LM3), que cresce espontaneamente em camundongos BALB/c, foi doada generosamente pela Dra. Elisa Bal de Kier Joffé do Instituto de Oncologia Angel H. Roffo, Buenos Aires, Argentina. As células foram cultivadas em meio MEM (Gibco®, USA), suplementado com $10 \%$ de soro fetal bovino (Gibco®, USA) e $4 \mu \mathrm{g} \mathrm{mL} \mathrm{m}^{-1}$ de gentamicina (Aldrich), a $37^{\circ} \mathrm{C}$ em atmosfera úmida com $5 \%$ de $\mathrm{CO}_{2}$. As células foram separadas por tripsina a $0,01 \%$ em EDTA $1 \mathrm{mmol} \mathrm{L}^{-1}$ (Gibcoß, EUA), realizando repiques três vezes por semana. As células aderentes viáveis foram determinadas pela contagem em câmara de Neubauer utilizando o corante azul de Tripan $(0,04 \%)$. Após a cultura, as células foram tratadas com diferentes concentrações dos compostos por $24 \mathrm{~h}$.

\section{Determinação da viabilidade celular}

Para avaliar a viabilidade celular da linhagem tumoral LM3 foi usado o ensaio com brometo de 3-(4,5-dimetiltiazol-2-il)-2-5-difeniltetrazólio (MTT). ${ }^{34}$ Em placas de 96 poços com fundo chato, foram plaqueadas, em triplicata, 5,0 x $10^{4}$ células/poço da linhagem tumoral em seu respectivo meio de cultura, para serem incubadas em estufa a $37^{\circ} \mathrm{C}$ e $5 \%$ de $\mathrm{CO}_{2}$. Após $24 \mathrm{~h}$, as células foram tratadas com os compostos e mantidas, novamente, em estufa por mais $24 \mathrm{~h}$. Em seguida, a viabilidade celular foi determinada adicionando a solução de MTT ( $5 \mathrm{mg} \mathrm{mL}^{-1}$ ) em cada poço e incubando as placas no escuro por $3 \mathrm{~h}$ a $37^{\circ} \mathrm{C}$ e $5 \%$ de $\mathrm{CO}_{2}$. Após esse período, foi adicionado isopropanol para solubilização dos cristais de formazan e a leitura de absorbância foi realizada em $540 \mathrm{~nm}$ em espectrofotômetro UV/visível para microplacas (Multiskan Ascent, Labsystems, Finlândia).

Os ensaios de citotoxicidade celular in vitro foram realizados com os ligantes livres (benzaldeidoxima e etilenotiouréia), com o complexo precursor (P) e com os complexos 1-3. Os compostos foram previamente diluídos em DMSO e posteriormente em meio de cultura, de tal modo que a concentração final de DMSO (1\%) não revelou nenhuma atividade citotóxica. Controles positivos e 


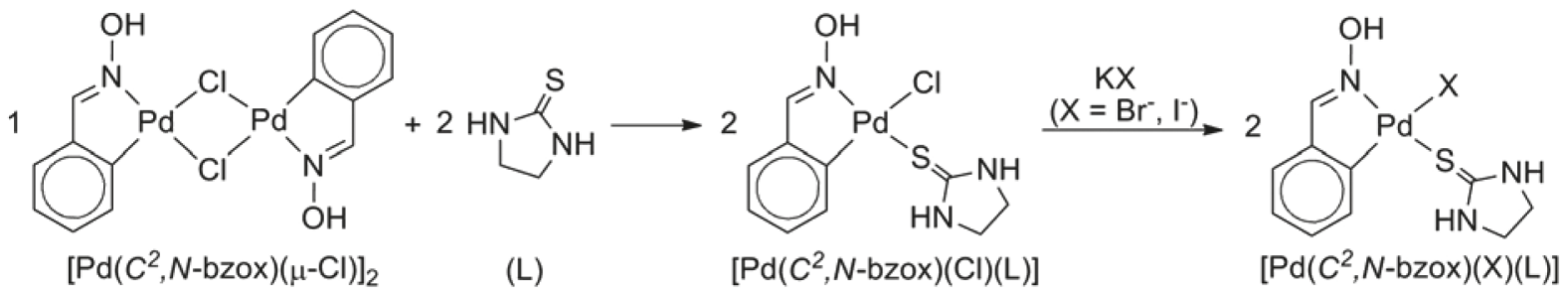

Figura 1. Esquema de síntese dos complexos $\left[\mathrm{Pd}\left(\mathrm{C}^{2}, \mathrm{~N}\right.\right.$-bzox $\left.)(\mathrm{X})(\mathrm{L})\right]\left\{\mathrm{X}=\mathrm{Cl}^{-}(\mathbf{1}), \mathrm{Br}\right.$ (2) e I (3) $\}$

Tabela 1. Análise elementar e temperatura de decomposição para os complexos 1-3 e precursor $\mathbf{P}$

\begin{tabular}{lccccccc}
\hline \multirow{2}{*}{ Compostos } & \multirow{2}{*}{$\left(^{\circ} \mathrm{C}\right)$} & \multicolumn{2}{c}{ \% Carbono } & \multicolumn{2}{c}{$\%$ Hidrogênio } & \multicolumn{2}{c}{$\%$ Nitrogênio } \\
\cline { 3 - 8 } & & Calc. & Obt. & Calc. & Obt. & Calc. & Obt. \\
\hline$\left[\operatorname{Pd}\left(C^{2}, N \text {-bzox }\right)(\mu-C l)\right]_{2}(\mathbf{P})$ & 231 & 32,0 & 32,1 & 2,31 & 2,11 & 5,25 & 5,19 \\
{$\left[\operatorname{Pd}\left(C^{2}, N\right.\right.$-bzox $\left.)(\mathrm{Cl})(\mathrm{L})\right](\mathbf{1})$} & 145 & 32,9 & 32,4 & 3,32 & 3,46 & 11,5 & 11,1 \\
{$\left[\operatorname{Pd}\left(C^{2}, N\right.\right.$-bzox $\left.)(\mathrm{Br})(\mathrm{L})\right](\mathbf{2})$} & 183 & 29,3 & 29,0 & 2,96 & 3,17 & 10,1 & 10,2 \\
{$\left[\operatorname{Pd}\left(C^{2}, N\right.\right.$-bzox $\left.)(\mathrm{I})(\mathrm{L})\right](\mathbf{3})$} & 194 & 26,3 & 26,7 & 2,65 & 2,60 & 9,22 & 9,72 \\
\hline
\end{tabular}

negativos foram incluídos em todos os experimentos. Os valores de $\mathrm{IC}_{50}$ (concentração necessária para inibir $50 \%$ da proliferação celular) foram determinados por meio da curva dose-resposta empregando o programa Microcal Origin Pro 8.0 ${ }^{\mathrm{TM}}$.

\section{RESULTADOS E DISCUSSÃO}

O complexo precursor $\left[\operatorname{Pd}\left(C^{2}, N \text {-bzox }\right)(\mu-\mathrm{Cl})\right]_{2}(\mathbf{P})$ foi preparado pela transciclometalação do $\left[\operatorname{Pd}\left(C^{2}, N-d m b a\right)(\mu-C l)\right]_{2}$ com a benzaldeidoxima (bzox). ${ }^{33} \mathrm{O}$ complexo $\left[\mathrm{Pd}\left(C^{2}, N\right.\right.$-bzox $\left.)(\mathrm{Cl})(\mathrm{L})\right](\mathbf{1})$ foi sintetizado pela reação de clivagem do complexo precursor com o ligante etilenotiouréia (L) na proporção molar 1:2, enquanto que os complexos $\left[\mathrm{Pd}\left(C^{2}, N\right.\right.$-bzox $\left.)(\mathrm{X})(\mathrm{L})\right]\left\{\mathrm{X}=\mathrm{Br}^{-}(\mathbf{2})\right.$ e $\left.\mathrm{I}^{-}(\mathbf{3})\right\}$ foram obtidos a partir do complexo 1 pela reação de substituição de haleto, como mostra a Figura 1.

Os complexos obtidos são sólidos estáveis à luz e ao ar e, com exceção do precursor, todos são solúveis em acetona gerando soluções com coloração amarelo-alaranjada. A Tabela 1 mostra os resultados de análise elementar e a temperatura de decomposição para os complexos 1-3 e para o precursor $\mathbf{P}$.

Os resultados da análise elementar estão em concordância com as fórmulas $\left[\operatorname{Pd}\left(C^{2}, N \text {-bzox }\right)(\mu-\mathrm{Cl})\right]_{2}$ e $\left[\operatorname{Pd}\left(C^{2}, N\right.\right.$-bzox $\left.)(\mathrm{X})(\mathrm{L})\right]$, condizendo com a estrutura binuclear proposta para o complexo $\mathbf{P}$ e mononuclear proposta para os complexos 1-3. Os complexos foram caracterizados por espectroscopia vibracional no infravermelho (IV) e espectroscopias de ressonância magnética nuclear (RMN) de ${ }^{1} \mathrm{H}$ e ${ }^{13} \mathrm{C}\left\{{ }^{1} \mathrm{H}\right\}$. Os resultados obtidos encontram-se a seguir.

\section{Espectroscopia vibracional no infravermelho}

As frequências vibracionais no infravermelho trazem informações importantes sobre o modo de coordenação dos ligantes ao centro metálico nos complexos. A Tabela 2 apresenta as principais absorções observadas nos espectros do precursor $\mathbf{P}$ e dos complexos 1-3.

As principais bandas atribuídas às oximas no infravermelho referem-se aos estiramentos das ligações $\mathrm{O}-\mathrm{H}, \mathrm{C}=\mathrm{N}, \mathrm{N}-\mathrm{O}$ e a deformação fora do plano das ligações $\mathrm{C}-\mathrm{H}$ de anéis aromáticos. ${ }^{35,36}$ Para a benzaldeidoxima (bzox), as bandas em 3186, 1634 e 984 $\mathrm{cm}^{-1}$ são atribuídas, respectivamente, aos modos vibracionais $\mathrm{vOH}$, $v \mathrm{C}=\mathrm{N}$ e $v \mathrm{NO}$, enquanto que a presença de duas bandas intensas em 755 e $691 \mathrm{~cm}^{-1}$, referentes ao modo vibracional $\gamma \mathrm{CH}$, indicam a estrutura monosubstituída do anel aromático da bzox..$^{35,37}$ No complexo precursor $\left[\operatorname{Pd}\left(C^{2}, N \text {-bzox }\right)(\mu-C l)\right]_{2}(\mathbf{P})$ o deslocamento das bandas
Tabela 2. Principais frequências vibracionais $\left(\mathrm{cm}^{-1}\right)$ para os complexos $\left[\operatorname{Pd}\left(C^{2}, N\right.\right.$-bzox $\left.)(\mathrm{X})(\mathrm{L})\right]\left\{\mathrm{X}=\mathrm{Cl}^{-}(\mathbf{1}), \operatorname{Br}(\mathbf{2}), \mathrm{I}^{-}(\mathbf{3})\right\}$ e $\left[\operatorname{Pd}\left(C^{2}, N \text {-bzox }\right)(\mu-\mathrm{Cl})\right]_{2}$ (P)

\begin{tabular}{ccccc}
\hline $\mathbf{P}$ & $\mathbf{1}$ & $\mathbf{2}$ & $\mathbf{3}$ & Atribuição \\
\hline $3417 F$ & $3400 \mathrm{om}$ & $*$ & $*$ & $v \mathrm{OH}$ \\
- & $3234 F$ & $3240 F$ & $*$ & $v \mathrm{NH}$ \\
$3053 f$ & $3052 f$ & $3048 f$ & $3043 f$ & $v \mathrm{CH}$ \\
- & $2888 f$ & $2225 f$ & $2883 f$ & $v \mathrm{CH}_{2}$ \\
$1623 f$ & $1629 f$ & $1628 f$ & $1621 f$ & $v \mathrm{C}=\mathrm{N}$ \\
- & $1534 F$ & $1533 F$ & $1529 F$ & $v \mathrm{CN}+\delta \mathrm{NH}$ \\
$1039 F$ & $1037 m F$ & $1034 m F$ & $1030 m$ & $v \mathrm{NO}$ \\
$747 F$ & $750 \mathrm{~m} F$ & $754 m F$ & $754 m F$ & $\gamma \mathrm{CH}$ \\
- & $913 f$ & $912 f$ & $911 f$ & $\gamma$ anel $+\mathrm{vC}=\mathrm{S}+\mathrm{vCN}$ \\
- & $498 m f$ & $501 m$ & $497 m$ & $v \mathrm{C}=\mathrm{S}$ \\
\hline
\end{tabular}

Abreviações: $F=$ forte; $f=$ fraco; $m=$ médio; $o m=$ ombro; $v=$ estiramento; $\gamma=$ deformação fora do plano; * banda mascarada

vOH em $216 \mathrm{~cm}^{-1}$, e vNO em $69 \mathrm{~cm}^{-1}$, para frequências vibracionais maiores, está em concordância com o esperado para os estiramentos dessas ligações quando a oxima se coordena ao centro metálico pelo nitrogênio imínico. ${ }^{36,38-40} \mathrm{As}$ bandas $\gamma \mathrm{CH}$ podem ser utilizadas como evidência para ortometalação. A presença de apenas uma banda intensa em $747 \mathrm{~cm}^{-1}$ para o complexo $\mathbf{P}$, em comparação a duas bandas intensas $\gamma \mathrm{CH}$ no ligante livre, corrobora com a ortodissubstituição do anel aromático da benzaldeidoxima. ${ }^{37,41}$

Os espectros no infravermelho dos complexos $\left[\mathrm{Pd}\left(C^{2}, N\right.\right.$-bzox $)(\mathrm{X})$ (L) $\left\{\mathrm{X}=\mathrm{Cl}^{-}(\mathbf{1}), \mathrm{Br}^{-}(\mathbf{2}), \mathrm{I}^{-}(\mathbf{3})\right\}$ apresentaram as bandas características da benzaldeidoxima ortometalada, além de bandas atribuídas ao ligante etilenotiouréia (L). A estrutura do L pode ser descrita por um hibrido de ressonância com a contribuição de três formas canônicas, a tiona (A) e a tiol (B/C) ${ }^{42}$ como mostrado na Figura 2.

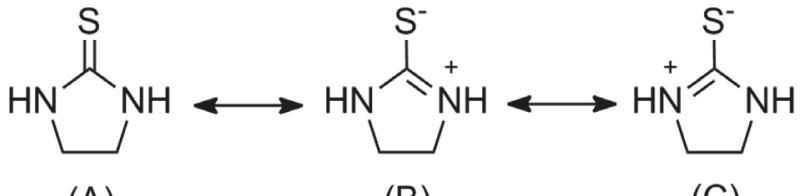

(A)

(B)

(C)

Figura 2. Formas canônicas da etilenotiouréia $(L)$ 
Para etilenotiouréia (L), as principais bandas no infravermelho referem-se aos estiramentos das ligações $\mathrm{N}-\mathrm{H}, \mathrm{C}-\mathrm{N}$ e C $=\mathrm{S} .{ }^{43} \mathrm{~A}$ etilenotiouréia pode se coordenar ao centro metálico tanto pelo átomo de enxofre quanto pelos átomos de nitrogênio. Se a coordenação ocorrer via átomo de enxofre, as formas canônicas $\mathrm{B}$ e C aumentam sua contribuição estrutural, promovendo um enfraquecimento da ligação $\mathrm{C}=\mathrm{S}$ e um fortalecimento da ligação C-N..$^{37,44}$ Nos complexos 1-3, a banda $v C=S\left(511 \mathrm{~cm}^{-1}\right.$, na etilenotiouréia livre $)$ encontra-se deslocada, cerca de $12 \mathrm{~cm}^{-1}$, para frequências vibracionais menores, enquanto que a banda $v C N\left(1522 \mathrm{~cm}^{-1}\right.$, na etilenotiouréia livre) encontra-se deslocada, cerca de $10 \mathrm{~cm}^{-1}$, para frequências maiores, sugerindo que a coordenação do ligante ocorreu via átomo de enxofre. Além disso, a presença da banda intensa $v \mathrm{NH}\left(3250 \mathrm{~cm}^{-1}\right.$ na etilenotiouréia livre) nos complexos confirma a presença do ligante neutro em sua estrutura. ${ }^{25}$

\section{Ressonância magnética nuclear}

Os espectros de RMN de ${ }^{1} \mathrm{H}$ dos complexos 1-3 exibem apenas um conjunto de sinais, o que confirma a presença de uma única espécie em solução. Os sinais dos átomos de hidrogênio e carbono foram atribuídos a partir das correlações observadas nos espectros de HMQC e HMBC e segue o esquema de numeração indicado na Figura 3.<smiles>ON1C=[C]c2ccccc2[Pb]1(Cl)SC1CCN1</smiles>

Figura 3. Numeração adotada para atribuição dos sinais de $R M N$ de ${ }^{l} \mathrm{H}$ e ${ }^{13} \mathrm{C}\left\{{ }^{1} \mathrm{H}\right\}$

No RMN de ${ }^{1} \mathrm{H}$, o aparecimento de apenas quatro sinais na região dos aromáticos, em torno de 7,32 (H3, dd), 7,28 (H6, dd), 7,09 (H4, td) e 7,02 ppm (H5, td) condiz com a presença do anel ortopaladado da bzox na estrutura dos complexos 1-3. Nos espectros de RMN de ${ }^{13} \mathrm{C}\left\{{ }^{1} \mathrm{H}\right\}$ dos complexos 1-3, o deslocamento do sinal de carbono do grupo imínico $(-\mathrm{HC}=\mathrm{N})$ de, aproximadamente, $10 \mathrm{ppm}$ para campo mais baixo sugere a coordenação da bzox pelo átomo de nitrogênio. Além disso, o surgimento de um sinal de carbono quartenário em 142,45 ppm, atribuído a C2 e deslocado para campo mais baixo em relação ao ligante livre $(126,55 \mathrm{ppm})$ confirma a presença do fragmento $C^{2}, N$-ortopaladado da bzox na estrutura dos complexos 1-3. . $^{32,45}$

A espectroscopia de RMN também confirma a coordenação do ligante etilenotiouréia via átomo de enxofre. Nos espectros de RMN de ${ }^{1} \mathrm{H}$ dos complexos 1-3, o deslocamento para campo mais baixo dos núcleos de hidrogênio $\mathrm{NH}$, em relação ao ligante livre, é atribuído a uma diminuição da densidade eletrônica ao redor do átomo de nitrogênio, decorrente da coordenação do ligante via átomo de enxofre, o que enfraquece a ligação $\mathrm{C}=\mathrm{S}$ enquanto fortalece a ligação $\mathrm{C}-\mathrm{N}$. Esse fato é confirmado pelo deslocamento do sinal ${ }^{13} \mathrm{C}=\mathrm{S}$ para campo mais alto, nos espectros de RMN de ${ }^{13} \mathrm{C}\left\{{ }^{1} \mathrm{H}\right\}$ dos complexos 1-3. ${ }^{21,25,44}$ Os dados de RMN de ${ }^{1} \mathrm{H} \mathrm{e}{ }^{13} \mathrm{C}\left\{{ }^{1} \mathrm{H}\right\}$ para os ligantes e o complexo $\mathbf{1}$ estão reunidos na Tabela 3 .

\section{Atividade citotóxica frente a linhagem tumoral LM3}

A atividade citotóxica dos complexos 1-3, do precursor $\mathbf{P}$ e dos ligantes livres (bzox e L) foi avaliada frente às células murinas de adenocarcinoma mamário (LM3). A Tabela 4 exibe os valores de $\mathrm{IC}_{50}$ obtidos em comparação com o da cisplatina e com o dos ciclopaladados do tipo $\left[\mathrm{Pd}\left(C^{2}, N\right.\right.$-dmba $\left.)(\mathrm{X})(\mathrm{tu})\right](\mathrm{X}=\mathrm{Cl}, \mathrm{Br}, \mathrm{I})$ frente a essa linhagem tumoral.

A benzaldeidoxima e a etilenotiouréia foram consideradas inativas, visto que apresentaram uma baixa citotoxicidade frente à linhagem tumoral. No entanto, todos os complexos mostram-se mais ativos, indicando o sinergismo na associação dos ligantes ao centro de paládio(II).

O complexo binuclear $\mathbf{P}$ mostrou-se menos ativo que os complexos mononucleares 1-3, que por sua vez, foram mais citotóxicos frente a LM3 que a cisplatina, destacando a importância da associação

Tabela 3. Dados obtidos por RMN de ${ }^{1} \mathrm{H} \mathrm{e}{ }^{13} \mathrm{C}\left\{{ }^{1} \mathrm{H}\right\}$ dos ligantes (bzox e L) em dmso- $d_{6}$, e do complexo 1 em acetona- $d_{6}$, em $500 \mathrm{MHz}$ a $25^{\circ} \mathrm{C}$, em ppm, atribuição, multiplicidade, [integral] e $J(\mathrm{~Hz})$

\begin{tabular}{|c|c|c|c|c|c|}
\hline \multirow{2}{*}{ Atribuição } & & \multicolumn{2}{|c|}{ bzox } & \multicolumn{2}{|c|}{$\left[\operatorname{Pd}\left(C^{2}, N\right.\right.$-bzox $\left.)(\mathrm{Cl})(\mathrm{L})\right]$} \\
\hline & & ${ }^{1} \mathrm{H}$ & $\delta{ }^{13} \mathrm{C}\left\{{ }^{1} \mathrm{H}\right\}$ & $\delta{ }^{1} \mathrm{H}$ & $\delta{ }^{13} \mathrm{C}\left\{{ }^{1} \mathrm{H}\right\}$ \\
\hline \multirow{8}{*}{ 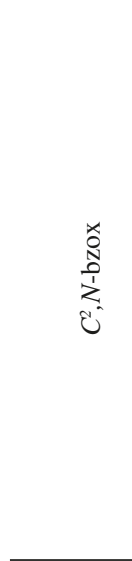 } & 1 & - & 133,20 & - & 142,45 \\
\hline & 2 & $\begin{array}{c}7,60 ; \mathrm{dd} ;[2 \mathrm{H}] \\
J_{23}=8,0 \text { e } J_{24}=2,2\end{array}$ & 126,55 & - & 150,47 \\
\hline & 3 & 7,42-7,38; m; [3H] & 126,55 & $\begin{array}{c}7,28 ; \mathrm{dd} ;[1 \mathrm{H}] \\
J_{34}=8,0 \text { e } J_{35}=1,5\end{array}$ & 131,67 \\
\hline & 4 & 7,42-7,38; m; [3H] & 129,37 & $\begin{array}{c}7,02 ; \mathrm{td} ;[1 \mathrm{H}] \\
J_{45}=J_{43}=8,0 \text { e } J_{46}=1,5\end{array}$ & 128,65 \\
\hline & 5 & 7,42-7,38; m; [3H] & 128,90 & $\begin{array}{c}7,09 ; \mathrm{td} ;[1 \mathrm{H}] \\
J_{54}=J_{56}=8,0 \text { e } J_{53}=1,5\end{array}$ & 125,42 \\
\hline & 6 & $\begin{array}{c}7,60 ; \mathrm{dd} ;[2 \mathrm{H}] \\
J_{65}=8,0 \text { e } J_{64}=2,2\end{array}$ & 129,37 & $\begin{array}{c}7,32 ; \mathrm{dd} ;[1 \mathrm{H}] \\
J_{65}=8,0 \text { e } J_{64}=1,5\end{array}$ & 127,22 \\
\hline & 7 & 8,$14 ; \mathrm{s} ;[1 \mathrm{H}]$ & 148,33 & 8,$22 ; \mathrm{s} ;[1 \mathrm{H}]$ & 157,39 \\
\hline & $\mathrm{OH}$ & 11,$20 ; \mathrm{s} ;[1 \mathrm{H}]$ & - & 10,$35 ; \mathrm{s} ;[1 \mathrm{H}]$ & - \\
\hline \multirow{3}{*}{ 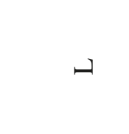 } & 1'e 2' & 3,$49 ; \mathrm{s} ;[4 \mathrm{H}]$ & 44,11 & 3,$92 ; \mathrm{s} ;[4 \mathrm{H}]$ & 45,78 \\
\hline & $\mathrm{NH}$ & 7,$95 ; \mathrm{s} ;[2 \mathrm{H}]$ & - & 8,$61 ; \mathrm{s} ;[2 \mathrm{H}]$ & - \\
\hline & $\mathrm{C}=\mathrm{S}$ & - & 183,45 & - & $*$ \\
\hline
\end{tabular}

Multiplicidade: $\mathrm{s}=$ singleto; $\mathrm{dd}=$ duplo dupleto; $\mathrm{td}=$ tripleto de dupleto. *Não detectado. 
Tabela 4. Atividade citotóxica $\left(\mathrm{IC}_{50}, \mu \mathrm{M}\right)$ dos complexos 1-3, do precursor $\mathbf{P}$ e dos ligantes livres em comparação a cisplatina e outros ciclopaladados frente a linhagem LM3

\begin{tabular}{lc}
\hline Compostos & LM3 \\
\hline$\left[\operatorname{Pd}\left(C^{2}, N \text {-bzox }\right)(\mu-C l)\right]_{2}(\mathbf{P})$ & $58,9 \pm 4,27$ \\
{$\left[\operatorname{Pd}\left(C^{2}, N\right.\right.$-bzox $\left.)(\mathrm{Cl})(\mathrm{L})\right](\mathbf{1})$} & $23,2 \pm 1,77$ \\
{$\left[\operatorname{Pd}\left(C^{2}, N\right.\right.$-bzox $\left.)(\mathrm{Br})(\mathrm{L})\right](\mathbf{2})$} & $19,9 \pm 0,61$ \\
{$\left[\operatorname{Pd}\left(C^{2}, N\right.\right.$-bzox $\left.)(\mathrm{I})(\mathrm{L})\right](\mathbf{3})$} & $18,2 \pm 2,87$ \\
Bzox & $>200$ \\
$\mathrm{~L}$ & $>200$ \\
Cisplatina $^{21}$ & $30,3 \pm 3,72$ \\
{$\left[\operatorname{Pd}\left(C^{2}, N \text {-dmba }\right)(\mathrm{Cl})(\mathrm{tu})\right]^{21}$} & $72,4 \pm 3,92$ \\
{$\left[\operatorname{Pd}\left(C^{2}, N \text {-dmba }\right)(\mathrm{Br})(\mathrm{tu})\right]^{21}$} & $29,6 \pm 0,18$ \\
{$\left[\operatorname{Pd}\left(C^{2}, N \text {-dmba }\right)(\mathrm{I})(\mathrm{tu})\right]^{20}$} & $14,4 \pm 1,92$ \\
\hline
\end{tabular}

do metalociclo ao ligante tiocarbonilado na atividade citotóxica dos complexos. Diferentemente do comportamento observado para os compostos do tipo $\left[\operatorname{Pd}\left(C^{2}, N\right.\right.$-dmba)(X)(tu) $]$, em que a atividade citotóxica dos compostos aumentou na sequência $\mathrm{I}>\mathrm{Br}>$ $\mathrm{Cl}^{20,21}$ os ciclopaladados 1-3 apresentaram níveis de citotoxicidade

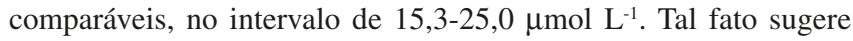
que a alteração do haleto na esfera de coordenação do paládio não produz efeito significativo na citotoxicidade, sendo plausível que os compostos 1-3 originem espécie(s) ativa(s) similar(es) no meio de cultura. De fato, Ryabov et al. ${ }^{32}$ evidenciaram que ciclopaladados do tipo $\left[\mathrm{Pd}\left(C^{2}, N\right.\right.$-bzox $\left.)(\mathrm{Cl})(\mathrm{L})\right]\left\{C^{2}, N\right.$-bzox = benzaldeidoxima e $\mathrm{L}=$ piridina $\}$ são suscetíveis à hidrolise em $\mathrm{pH}$ de 3 a 10 , resultando em espécies do tipo $\left[\mathrm{Pd}\left(C^{2}, N\right.\right.$-bzox $\left.)(\mathrm{OH})(\mathrm{L})\right]$ e $\left[\mathrm{Pd}\left(C^{2}, N\right.\right.$-bzox $)\left(\mathrm{OH}_{2}\right)$ $(\mathrm{L})]^{+}$. Tendo em vista as conclusões do trabalho de Ryabov, é muito provável que os compostos 1-3 também tenham um comportamento análogo em solução, entretanto, são necessários mais estudos para elucidar a natureza dessas espécies em solução.

\section{CONCLUSÃO}

Esse trabalho descreveu a síntese e caracterização espectroscópica de uma série inédita de ciclopaladados contendo benzaldeidoxima ortometalada de fórmula $\left[\mathrm{Pd}\left(C^{2}, N\right.\right.$-bzox $\left.)(\mathrm{X})(\mathrm{L})\right]\left\{\mathrm{X}=\mathrm{Cl}^{-}(\mathbf{1}), \mathrm{Br}(\mathbf{2})\right.$, $\mathrm{I}^{-}(\mathbf{3}) ; \mathrm{L}=$ etilenotiouréia $\}$. Os produtos foram obtidos com sucesso a partir da clivagem de dímero precursor pela etilenotiouréia e posterior reação de metátese do ligante cloro pelo haleto apropriado. A estrutura mononuclear dos compostos e a coordenação do ligante sulfurado foram evidenciadas por técnicas espectroscópicas em solução (RMN) e no estado sólido (infravermelho).

Os compostos ciclopaladados obtidos neste trabalho exibiram índices de citotoxicidade semelhantes $\left(15,3-25,0 \mu \mathrm{mol} \mathrm{L}^{-1}\right)$ frente à linhagem de adenocarcinoma mamário murino LM3, sugerindo que espécies ativas similares sejam produzidas durante a incubação desses compostos no meio de cultura. Cabe destacar que todos os compostos se mostraram mais citotóxicos que a cisplatina $\left(30,3 \pm 3,72 \mu \mathrm{mol} \mathrm{L}^{-1}\right)$. Comparando-se os resultados desse trabalho com aqueles descritos para a família $\left[\operatorname{Pd}\left(C^{2}, N-d m b a\right)(\mathrm{X})(\mathrm{L})\right]$, fica evidente que a substituição da $N, N$-dimetilbenzilamina pela benzaldeidoxima ortometalada teve um papel importante na diferença do padrão de citotoxicidade dessas duas séries de ciclopaladados, o que poderá nortear a síntese de futuros análogos contendo oximas ortometaladas.

\section{MATERIAL SUPLEMENTAR}

Espectros no infravermelho e ressonância magnética nuclear de ${ }^{1} \mathrm{H}$ e ${ }^{13} \mathrm{C}\left\{{ }^{1} \mathrm{H}\right\}$ dos complexos e dos ligantes (bzox e L) relatados neste trabalho estão disponíveis para livre acesso em http://quimicanova. sbq.org.br.

\section{AGRADECIMENTOS}

Agradecemos à Dra. E. B. K. Joffé do Instituto de Oncologia Angel H. Roffo pela doação da linhagem celular de tumor mamário murino LM3. Agradecemos, também, a M. C. P. Placeres pelo suporte técnico nos ensaios biológicos. Esta pesquisa foi apoiada pela FAPESP (proc. 2012/15486-3 e 2016/177115), pelo CNPq (proc. 422105/2016-3) e pela CAPES.

\section{REFERÊNCIAS}

1. Colotti, G.; Ilari, A.; Boffi, A.; Morea, V.; Mini-Rev. Med. Chem. 2013, 13, 211.

2. Drewry, J. A.; Gunning, P. T.; Coord. Chem. Rev. 2011, 255, 459.

3. Rijt, S. H. V.; Sadler, P. J.; Drug Discovery Today 2010, 14, 1089.

4. Bruijnincx, P. C. A.; Sadler, P. J.; Curr. Opin. Chem. Biol. 2008, 12, 197.

5. Sadler, P. J.; Platinum Met. Rev. 2008, 52, 21.

6. Cohen, S. M.; Curr. Opin. Chem. Biol. 2007, 11, 115.

7. Albert, J.; Granell, J.; Qadir, R.; Quirante, J.; Calvis, C.; Messeguer, R.; Badía, J.; Baldomà, L.; Bardia, M. F.; Calvet, T.; Organometallics 2014, 33, 7284.

8. Frezza, M.; Hindo, S.; Chen, D.; Davenport, A.; Schmitt, S.; Tomco, D.; Ping Dou, Q.; Curr. Pharm. Des. 2010, 16, 1813.

9. Medici, S.; Peana, M.; Nurchi, V. M.; Lachowicz, J. I.; Crisponi, G.; Zoroddu, M. A.; Coord. Chem. Rev. 2015, 284, 329.

10. Gielen, M.; Tiekink, E. R. T.; Metallotherapeutic Drugs and MetalBased Diagnostic Agents: The Use of Metals in Medicine, $1^{\text {st }}$ ed., John Wiley \& Sons Ltd: England, 2007.

11. Garoufis, A.; Hadjikakou, S. K.; Hadjiliadis, N.; Coord. Chem. Rev. 2009, 253, 1384.

12. Bechara, A.; Barbosa, C. M. V.; Paredes-Gamero, E. J.; Garcia, D. M.; Silva, L. S.; Matsuo, A. L.; Nascimento, F. D.; Rodrigues, E. G.; Caires, A. C. F.; Smaili, S. S.; Bincoletto, C.; Eur. J. Med. Chem. 2014, 79, 24.

13. Alam, M. N.; Huq, F.; Coord. Chem. Rev. 2016, 316, 36.

14. Gigli, R.; Pereira, G. J. S.; Antunes, F.; Bechara, A.; Garcia, D. M.; Spindola, D. G.; Jasiulionis, M. G.; Caires, A. C. F.; Smaili, S. S.; Bincoletto, C.; Eur. J. Med Chem. 2016, 107, 245.

15. Caires, A. C. F.; Anti-Cancer Agents Med. Chem. 2007, 7, 484.

16. Cutillas, N.; Yellol, G. S.; De Haro, C.; Vicente, C.; Rodríguez, V.; Ruiz, J.; Coord. Chem. Rev. 2013, 257, 2784.

17. Omae, I.; Coord. Chem. Rev. 2004, $280,84$.

18. Caires, A. C. F.; Mauro, A. E.; Quim. Nova 1996, 19, 59.

19. Moraes, V. W. R.; Caires, A. C. F.; Paredes-Gamero, E. J.; Rodrigues, T.; Cell Death Dis. 2013, 4, e658.

20. Moro, A. C.; da Cunha, G. A.; de Souza, R. F. F.; Mauro, A. E.; Netto, A. V. G.; Carlos, I. Z.; Resende, F. A.; Varanda, E. A.; Pavan, F. R.; Leite, C. Q. F.; Med. Chem. Res. 2015, 24, 2879.

21. Moro, A. C.; Mauro, A. E.; Netto, A. V. G.; Ananias, S. R.; Quilles, M. B.; Carlos, I. Z., Pavan, F. R.; Leite, C. Q. F.; Horner, M.; Eur. J. Med. Chem. 2009, 44, 4611.

22. Higgins, J. D.; Neely, L.; Fricker, S.; Matthey, J.; J. Inorg. Biochem. 1993, 49, 149.

23. Hoffman, R. V.; Organic Chemistry: An Intermediate Text, $2^{\text {nd }}$ ed., Wiley: Hoboken, 2004.

24. Alacid, E.; Alonso, D. A.; Botella, L.; Nájera, C.; Pacheco, M. C.; Chem. Rec. 2006, 6, 117.

25. Moura, T. R.; Cavalcanti, S. L.; de Godoy, P. R. D. V.; Sakamoto-Hojo, E. T.; Rocha, F. V.; de Almeida, E. T.; Deflon, V. M.; Mauro, A. E.; Netto, A. V. G.; Trans. Met. Chem. 2017, 42, 565. 
26. Silva, C.; Ribeiro, L. B.; Furuno, C. C.; da Cunha, G. A.; de Souza, R. F. F.; Netto, A. V. G.; Mauro, A. E.; Frem, R. C. G.; Fernandes, J. A.; Paz, F. A. A.; Marino, L. B.; Pavan, F. R.; Leite, C. Q. F.; Polyhedron 2015, $100,10$.

27. Barra, C. V.; Rocha, F. V.; Morel, L.; Gautier, A.; Garrido, S. S.; Mauro, A. E.; Frem, R. C. G.; Netto, A. V. G.; Inorg. Chim. Acta 2016, 446, 54.

28. Rocha, F. V.; Barra, C. V.; Garrido, S. S.; Manente, F. A.; Carlos, I. Z.; Ellena, J.; Fuentes, A. S. C.; Gautier, A.; Morel, L.; Mauro, A. E.; Netto, A. V. G.; J. Inorg. Biochem. 2016, 159, 165.

29. Cope, A. C.; Friedrich, E. C.; J. Am. Chem. Soc. 1968, 90, 909.

30. Maassarani, F.; Pfeffer, M.; Borgne, G. L.; Organometallics 1987, 6, 2029.

31. Lucca Neto, V. A.; Mauro, A. E.; Caires, A. C. F.; Ananias, S. R.; de Almeida, E. T.; Polyhedron 1998, 18, 413

32. Ryabov, A. D.; Kazankov, G. M.; Yatsimirsky, A. K.; Kuz'mina, L. G.; Burtseva, O. Y.; Dvortsova, N. V.; Polyakov, V. A.; Inorg. Chem. 1992, 31, 3083.

33. Selvakumar, K.; Vancheesan, S.; Varghese, B.; Polyhedron 1997, 16 , 2257.

34. Mosmann, T.; J. Immunol. Methods 1983, 65, 55.

35. Palm, A.; Werbin, H.; Can. J. Chem. 1953, 31, 1004.
36. Kaya, Y.; Icsel, C.; Yilmaz, V. T.; Buyukgungor, O.; J. Organomet. Chem. 2014, 752, 83.

37. Socrates, G.; Infrared and Raman characteristic group frequencies. Infrared and Raman characteristic group frequencies, $3^{\text {rd }}$ ed., John Wiley \& Sons Ltd: Chichester, 2004.

38. Keeney, M. E.; Osseo-Asare, K.; Woode, K. A.; Coord. Chem. Rev. 1984, 59, 141

39. Hiroshi, O.; Kyoji, M.; Kunio, N.; Bull. Chem. Soc. Jpn. 1970, 43, 3480.

40. Bozza, G. F.; de Farias, R. L.; de Souza, R. F. F.; Rocha, F. V.; Barra, C. V.; Deflon, V. M.; de Almeida, E. T.; Mauro, A. E.; Netto, A. V. G.; J. Mol. Struct. 2019, 1175, 195.

41. Nakamoto, K.; Infrared and Raman spectra of inorganic and coordination compounds, $5^{\text {th }}$ ed., John Wiley \& Sons Ltd: New York, 1997.

42. Wheatley, P. J.; Acta Crystallogr. 1953, 6, 369.

43. Dwarakanath, K.; Sathyanarayana, D. N.; Bull. Chem. Soc. Jpn. 1979, $52,2699$.

44. Nadeem, S.; Rauf, M. K.; Bolte, M.; Ahmad, S.; Tirmizi, S. A.; Asma, M.; Hameed, A.; Trans. Met. Chem. 2010, 35, 555.

45. Abellan-Lopez, A.; Chicote, M.-T.; Bautista, D.; Vicente, J.; Dalton Trans. 2014, 43, 592. 